\title{
The electromagnetic multipole moments of the possible charm-strange pentaquarks in light-cone QCD
}

\author{
K. Azizi ${ }^{1,2, a}$, U. Özdem ${ }^{1, b}$ \\ ${ }^{1}$ Department of Physics, Dogus University, Acibadem-Kadikoy, 34722 Istanbul, Turkey \\ ${ }^{2}$ School of Physics, Institute for Research in Fundamental Sciences (IPM), P.O. Box 19395-5531, Tehran, Iran
}

Received: 17 July 2018 / Accepted: 24 August 2018 / Published online: 31 August 2018

(C) The Author(s) 2018

\begin{abstract}
We investigate the electromagnetic properties of possible charm-strange pentaquarks in the framework of the light-cone QCD sum rule using the photon distribution amplitudes. In particular, by calculating the corresponding electromagnetic form factors defining the radiative transitions under consideration we estimate the magnetic dipole and electric quadrupole moments of the pentaquark systems of a charm, an anti-strange and three light quarks. We observe that the values of magnetic dipole moments are considerably large, however, the quadrupole moments are very small. Any future measurements of the electromagnetic parameters under consideration and comparison of the obtained data with the theoretical predictions can shed light on the quark-gluon organization as well as the nature of the pentaquarks.
\end{abstract}

\section{Introduction}

Although the existence of the exotic states was predicted many decades ago by Jaffe [1], this subject has experienced two revolutions in the last two decades. The first one was the discovery of the famous X(3872) tetraquark state by Belle experiment [2] in 2003. The second revolution was in 2015 when the LHCb Collaboration announced the observation of the hidden-charmed $P_{c}^{+}(4380)$ and $P_{c}^{+}(4450)$ pentaquarks with the spin-parities $J^{P}=\frac{3}{2}^{-}$and $\frac{5}{2}^{+}$, respectively [3]. Now we have many exotic states discovered via different experiments. For more information on the experimental and theoretical progresses on the features of these new particles see for instance Refs. [4-16]. Despite a lot of the experimental and theoretical efforts, since the discovery of the first exotic state in 2003 , on the physical properties of the non-conventional or exotic states, their internal quark-gluon organization, nature and quantum numbers are not well-

\footnotetext{
a e-mail: kazizi@dogus.edu.tr

be-mail: uozdem@dogus.edu.tr
}

established and there are many questions to be answered. The spectroscopic parameters of these states have been widely investigated both in theory and experiment. Many suggestions on the internal quark structure of the exotic states give consistent mass results with the experimental data. This prevents us to have exact assignments on the internal structure, nature and quantum numbers of the exotic states [17-20]. Hence, we need move investigations on the fundamental interactions of these states with each other and other known particles. Among these interactions are the electromagnetic interactions of these states and their radiative decays. Analysis of the electromagnetic and multipole moments of the exotic states can help us get valuable knowledge about the electromagnetic properties of these states, the charge distributions inside them, their charge radius and geometric shapes and finally their internal substructure.

As we mentioned above, the electromagnetic multipole moments are straight-forwardly connected with the charge and current distributions in the particles and these observables contain important information on the internal spatial quarks and gluons distributions of the particles. Their sign and magnitude encode valuable information on shape, structure and size of hadrons. There exist a lot of studies in the literature in which the electromagnetic properties of conventional hadrons are studied and electromagnetic multipole moments are obtained, but unluckily our knowledge on the electromagnetic multipole moments of the non-conventional hadrons are very limited. There exist only few studies in the literature devoted to the study of the electromagnetic multipole moments of the exotic states [21-34]. Theoretical works can play important roles in this respect since direct experimental information about the electromagnetic multipole moments of exotic particles is very limited. In this study, the electromagnetic multipole moments of the charm-strange pentaquark states (hereafter we will denote these states as $P_{c \bar{s}}$ ) are extracted by using the diquark-diquark-antiquark picture in the framework of the light-cone QCD sum rule (LCSR) 
(for more about this method see, e.g., [35-37] and references therein). This method has already been successfully applied to investigate the dynamical and statical properties of hadrons for many years such as, coupling constants, form factors, masses and electromagnetic multipole moments. In the LCSR, the features of the particles under investigations are defined based on the light-cone distribution amplitudes (DAs) that determine the matrix elements of the nonlocal operators between vacuum and corresponding particle states. Therefore, any uncertainty in these parameters affects the predictions on the electromagnetic multipole moments.

The rest part of the paper is coordinated in the following way: In Sect. 2, we present the result for the $P_{c \bar{s}}$ pentaquarks electromagnetic multipole moments in the LCSR method. Section 3 is devoted to the numerical analysis of the obtained sum rules. Section 4 includes our concluding remarks. The QCD sum rules of the electromagnetic form factors entering the expressions of the magnetic dipole and electric quadrupole moments are collected in the Appendix.

\section{Formalism}

In order to determine the electromagnetic multipole moments in the framework of the LCSR, we take into consideration the following two-point correlation function:

$$
\Pi_{\mu \nu}(q)=i \int d^{4} x e^{i p \cdot x}\left\langle 0\left|\mathcal{T}\left\{J_{\mu}(x) \bar{J}_{v}(0)\right\}\right| 0\right\rangle_{\gamma},
$$

where $\gamma$ means the external electromagnetic field, $J_{\mu}$ is the interpolating current of $P_{c \bar{s}}$ pentaquark with spin- $\frac{3}{2}$. In the diquark-diquark-antiquark picture, it can be written as [38]

$$
\begin{aligned}
J_{\mu}(x)= & \varepsilon^{a b c} \varepsilon^{a d e} \varepsilon^{b f g} \\
& \times\left[q_{1}^{d^{T}}(x) C \gamma_{5} q_{2}^{e}(x) q_{3}^{f^{T}}(x) C \gamma_{\mu} c^{g}(x) C \bar{s}^{c^{T}}(x)\right],
\end{aligned}
$$

where $q_{1}, q_{2}, q_{3}$ are $\mathrm{u}, \mathrm{d}$ and/or s-quark, $C$ is the charge conjugation operator; and $a, b \ldots$ represent color indices.

According to the philosophy of the QCD sum rules, the correlator, given in Eq. (1), can be calculated in two ways: (1) In terms of hadron parameters such as the masses, residues and the coupling constants, known as hadronic representation; (2) in terms of the quark-gluon parameters and using the photon DAs which include all nonperturbative dynamics, known as QCD representation. Then equating these two different representations of the correlation function to each other by the help of the quark-hadron duality assumption gives us the desired sum rules. In order to suppress the contributions of the higher states and continuum we apply Borel transformation, and continuum subtraction to both sides of the acquired QCD sum rules.
We start to compute the correlation function in terms of hadronic degrees of freedom including the physical properties of the particles under consideration. For this purpose, we insert an intermediate set of $P_{c \bar{s}}$ pentaquark into the correlation function. Consequently, we get

$$
\begin{aligned}
\Pi_{\mu \nu}^{H a d}(p, q)= & \frac{\left\langle 0\left|J_{\mu}\right| P_{c \bar{s}}(p)\right\rangle}{\left[p^{2}-m_{P_{c \bar{s}}}^{2}\right]}\left\langle P_{c \bar{s}}(p) \mid P_{c \bar{s}}(p+q)\right\rangle_{\gamma} \\
& \times \frac{\left\langle P_{c \bar{s}}(p+q)\left|\bar{J}_{\nu}\right| 0\right\rangle}{\left[(p+q)^{2}-m_{P_{c \bar{s}}}^{2}\right]},
\end{aligned}
$$

The matrix elements in Eq. (3) are described as [39,40],

$$
\begin{aligned}
& \left\langle 0\left|J_{\mu}\right| P_{c \bar{s}}(p, s)\right\rangle=\lambda_{P_{c \bar{s}}} u_{\mu}(p, s), \\
& \left\langle P_{c \bar{s}}(p) \mid P_{c \bar{s}}(p+q)\right\rangle_{\gamma} \\
& =-e \bar{u}_{\mu}(p)\left\{F_{1}\left(q^{2}\right) g_{\mu \nu} \phi\right. \\
& \quad-\frac{1}{2 m_{P_{c \bar{s}}}}\left[F_{2}\left(q^{2}\right) g_{\mu \nu}+F_{4}\left(q^{2}\right) \frac{q_{\mu} q_{\nu}}{\left(2 m_{P_{c \bar{s}}}\right)^{2}}\right] \notin \phi \\
& \left.\quad+F_{3}\left(q^{2}\right) \frac{1}{\left(2 m_{P_{c \bar{s}}}\right)^{2}} q_{\mu} q_{\nu} \notin\right\} u_{\nu}(p+q),
\end{aligned}
$$

where $\varepsilon$ and $\mathrm{q}$ are the polarization vector and momentum of the photon, respectively, $\lambda_{P_{c \bar{s}}}$ denotes the residue and $u_{\mu}(p, s)$ is the Rarita-Schwinger spinor of $P_{c \bar{s}}$ pentaquarks. Summation on spins of $P_{c \bar{s}}$ pentaquark is performed as:

$$
\begin{aligned}
& \sum_{s} u_{\mu}(p, s) \bar{u}_{v}(p, s)=-\left(\not p+m_{P_{c \bar{s}}}\right) \\
& \quad \times\left[g_{\mu \nu}-\frac{1}{3} \gamma_{\mu} \gamma_{\nu}-\frac{2 p_{\mu} p_{\nu}}{3 m_{P_{c \bar{s}}}^{2}}+\frac{p_{\mu} \gamma_{\nu}-p_{\nu} \gamma_{\mu}}{3 m_{P_{c \bar{s}}}}\right] .
\end{aligned}
$$

In principle, it is possible to acquire the final form of the hadronic representation of the correlator using the above equations, but we encounter with two problems: not all Lorentz structures are independent and the correlator can include not only the spin- $3 / 2$ contributions but also the contributions from the spin-1/2 particles, which must be removed. To eliminate the spin-1/2 contributions and acquire only independent structures in the correlator, we order the Dirac matrices as $\gamma_{\mu} \not p \phi \phi \gamma_{\nu}$ and remove terms starting with $\gamma_{\mu}$, and ending with $\gamma_{\nu}$ and those which are proportional to $p_{\mu}$ and $p_{\nu}$ [41]. This procedure eliminates the spin- $\frac{1}{2}$ pollutions. Consequently, using Eqs. (3) and (4) for hadronic side we get,

$$
\begin{aligned}
& \Pi_{\mu \nu}^{H a d}(p, q)=-\frac{\lambda_{P_{c \bar{s}}}^{2}}{\left[(p+q)^{2}-m_{P_{c \bar{s}}}^{2}\right]\left[p^{2}-m_{P_{c \bar{s}}}^{2}\right]} \\
& \times\left[-g_{\mu \nu} \not p \phi \phi F_{1}\left(q^{2}\right)+m_{P_{c \bar{s}}} g_{\mu \nu} \phi \phi F_{2}\left(q^{2}\right)+\frac{F_{3}\left(q^{2}\right)}{4 m_{P_{c \bar{s}}}} q_{\mu} q_{\nu} \phi \phi\right. \\
& \left.+\frac{F_{4}\left(q^{2}\right)}{4 m_{P_{c \bar{s}}}^{3}}(\varepsilon \cdot p) q_{\mu} q_{\nu} \not p \phi+\text { other independent structures }\right] .
\end{aligned}
$$


The magnetic dipole $\left(G_{M}\left(q^{2}\right)\right)$, electric quadrupole $\left(G_{Q}\left(q^{2}\right)\right)$, and magnetic octupole $\left(G_{O}\left(q^{2}\right)\right)$, form factors are described in terms of the form factors $F_{i}\left(q^{2}\right)$ as $[39,40]$ :

$$
\begin{aligned}
G_{M}\left(q^{2}\right)= & {\left[F_{1}\left(q^{2}\right)+F_{2}\left(q^{2}\right)\right]\left(1+\frac{4}{5} \lambda\right) } \\
& -\frac{2}{5}\left[F_{3}\left(q^{2}\right)+F_{4}\left(q^{2}\right)\right] \lambda(1+\lambda), \\
G_{Q}\left(q^{2}\right)= & {\left[F_{1}\left(q^{2}\right)-\lambda F_{2}\left(q^{2}\right)\right] } \\
& -\frac{1}{2}\left[F_{3}\left(q^{2}\right)-\lambda F_{4}\left(q^{2}\right)\right](1+\lambda) . \\
G_{O}\left(q^{2}\right)= & {\left[F_{1}\left(q^{2}\right)+F_{2}\left(q^{2}\right)\right] } \\
& -\frac{1}{2}\left[F_{3}\left(q^{2}\right)+F_{4}\left(q^{2}\right)\right](1+\lambda),
\end{aligned}
$$

where $\lambda=-\frac{q^{2}}{4 m_{P_{c \bar{s}}}^{2}}$. At $q^{2}=0$, the electromagnetic multipole form factors are acquired in terms of the functions $F_{i}(0)$ as:

$$
\begin{aligned}
G_{M}(0) & =F_{1}(0)+F_{2}(0), \\
G_{Q}(0) & =F_{1}(0)-\frac{1}{2} F_{3}(0), \\
G_{O}(0) & =F_{1}(0)+F_{2}(0)-\frac{1}{2}\left[F_{3}(0)+F_{4}(0)\right] .
\end{aligned}
$$

The magnetic dipole, $\left(\mu_{P_{c \bar{s}}}\right)$, electric quadrupole $\left(Q_{P_{c \bar{s}}}\right)$ and magnetic octupole moments $\left(O_{P_{c s}}\right)$ are described as follows,

$$
\begin{aligned}
\mu_{P_{c \bar{s}}} & =\frac{e}{2 m_{P_{c \bar{s}}}} G_{M}(0), \\
Q_{P_{c \bar{s}}} & =\frac{e}{m_{P_{c \bar{s}}}^{2}} G_{Q}(0), \\
O_{P_{c \bar{s}}} & =\frac{e}{2 m_{P_{c \bar{s}}}^{3}} G_{O}(0) .
\end{aligned}
$$

In present work we derive sum rules for the form factors $F_{i}\left(q^{2}\right)$ then in numerical analyses we will use the above relations to extract the values of the multipole moments using the sum rules for the form factors. The final form of the hadronic side in terms of the selected structures in momentum space is:

$$
\begin{aligned}
\Pi_{\mu \nu}^{H a d}(p, q)= & \Pi_{1}^{\text {Had }} g_{\mu \nu} \not p \phi \phi+\Pi_{2}^{H a d} g_{\mu \nu} \phi \phi+\Pi_{3}^{H a d} q_{\mu} q_{\nu} \phi \phi \\
& +\Pi_{4}^{H a d}(\varepsilon \cdot p) q_{\mu} q_{\nu} \not p q+\cdots,
\end{aligned}
$$

where $\Pi_{i}^{\text {Had }}$ are functions of the form factors $F_{i}\left(q^{2}\right)$ and other hadronic parameters; and ... represents other independent structures.

In the deep Euclidean region, the correlation function can also be computed in terms of quark-gluon fields as well as the photon DAs. Using expressions of interpolating currents and contracting all quark pairs, we get the following expression for the correlation function:

$$
\begin{aligned}
& \Pi_{\mu \nu}^{Q C D}(p, q)=i \varepsilon^{a b c} \varepsilon^{a^{\prime} b^{\prime} c^{\prime}} \varepsilon^{a d e} \varepsilon^{a^{\prime} d^{\prime} e^{\prime}} \varepsilon^{b f g} \varepsilon^{b^{\prime} f^{\prime} g^{\prime}} \\
& \times \int d^{4} x e^{i p \cdot x}\langle 0| \widetilde{S}_{s}^{c^{\prime} c}(-x)\left\{\operatorname{Tr}\left[\gamma_{5} \widetilde{S}_{c}^{g g^{\prime}}(x) \gamma_{5} S_{q_{3}}^{f f^{\prime}}(x)\right]\right. \\
& \times \operatorname{Tr}\left[\gamma_{\nu} \widetilde{S}_{q_{2}}^{e e^{\prime}}(x) \gamma_{\mu} S_{q_{1}}^{d d^{\prime}}(x)\right]-\operatorname{Tr}\left[\gamma_{5} \widetilde{S}_{c}^{g g^{\prime}}(x) \gamma_{5} S_{q_{3}}^{f f^{\prime}}(x)\right] \\
& \times \operatorname{Tr}\left[\gamma_{\nu} \widetilde{S}_{q_{1} q_{2}}^{d e^{\prime}}(x) \gamma_{\mu} S_{q_{2} q_{1}}^{e d^{\prime}}(x)\right]-\operatorname{Tr}\left[\gamma_{5} \widetilde{S}_{c}^{g g^{\prime}}(x) \gamma_{5} S_{q_{3} q_{1}}^{f d^{\prime}}(x)\right. \\
& \left.\times \gamma_{\nu} \widetilde{S}_{q_{2}}^{e e^{\prime}}(x) \gamma_{\mu} \widetilde{S}_{q_{1} q_{3}}^{d f^{\prime}}(x)\right]-\operatorname{Tr}\left[\gamma_{5} \widetilde{S}_{c}^{g g^{\prime}}(x) \gamma_{5} S_{q_{3} q_{2}}^{f e^{\prime}}(x)\right. \\
& \left.\times \gamma_{\nu} \widetilde{S}_{q_{1}}^{d^{\prime}}(x) \gamma_{\mu} \widetilde{S}_{q_{2} q_{3}}^{e f^{\prime}}(x)\right]+\operatorname{Tr}\left[\gamma_{5} \widetilde{S}_{c}^{g g^{\prime}}(x) \gamma_{5} S_{q_{3} q_{1}}^{f d^{\prime}}(x)\right. \\
& \left.\times \gamma_{\nu} \widetilde{S}_{q_{1} q_{2}}^{d e^{\prime}}(x) \gamma_{\mu} \widetilde{S}_{q_{3} q_{2}}^{e f^{\prime}}(x)\right]+\operatorname{Tr}\left[\gamma_{5} \widetilde{S}_{c}^{g g^{\prime}}(x) \gamma_{5} S_{q_{3} q_{2}}^{f e^{\prime}}(x)\right. \\
& \left.\left.\times \gamma_{\nu} \widetilde{S}_{q_{2} q_{1}}^{e d^{\prime}}(x) \gamma_{\mu} \widetilde{S}_{q_{1} q_{3}}^{d f^{\prime}}(x)\right]\right\}|0\rangle_{\gamma},
\end{aligned}
$$

where $\widetilde{S}_{c(q)}^{i j}(x)=C S_{c(q)}^{i j \mathrm{~T}}(x) C$ and $S_{q_{i} q_{j}}$ exists when $q_{i}=q_{j}$ but it vanishes when $q_{i} \neq q_{j}$.

The quark propagators $S_{q}(x)$ and $S_{c}(x)$ are given as [42]

$$
\begin{aligned}
S_{q}(x)= & S_{q}^{\text {free }}-\frac{\bar{q} q}{12}\left(1-i \frac{m_{q} \not x}{4}\right)-\frac{\bar{q} \sigma \cdot G q}{192} x^{2}\left(1-i \frac{m_{q} \chi x}{6}\right) \\
& -\frac{i g_{s}}{32 \pi^{2} x^{2}} G^{\mu \nu}(x)\left[k \sigma_{\mu \nu}+\sigma_{\mu \nu} x\right],
\end{aligned}
$$

and

$$
\begin{aligned}
& S_{c}(x)=S_{c}^{f r e e}-\frac{g_{s} m_{c}}{16 \pi^{2}} \int_{0}^{1} d v G^{\mu \nu}(v x) \\
& \times\left[\left(\sigma_{\mu \nu} \not x+\not \chi \sigma_{\mu \nu}\right) \frac{K_{1}\left(m_{c} \sqrt{-x^{2}}\right)}{\sqrt{-x^{2}}}+2 \sigma^{\mu \nu} K_{0}\left(m_{c} \sqrt{-x^{2}}\right)\right],
\end{aligned}
$$

where

$$
\begin{aligned}
& S_{q}^{\text {free }}(x)=i \frac{\not x}{2 \pi^{2} x^{4}}-\frac{m_{q}}{4 \pi^{2} x^{2}}, \\
& S_{c}^{\text {free }}(x)=\frac{m_{c}^{2}}{4 \pi^{2}}\left[\frac{K_{1}\left(m_{c} \sqrt{-x^{2}}\right)}{\sqrt{-x^{2}}}+i \frac{\not x K_{2}\left(m_{c} \sqrt{-x^{2}}\right)}{\left(\sqrt{-x^{2}}\right)^{2}}\right],
\end{aligned}
$$

with $K_{i}$ being the modified Bessel functions of the second kind.

The correlation function includes short distance (perturbative), and long distance (nonperturbative) contributions. In the first part, the propagator of the quark interacting with the photon perturbatively is replaced by

$S^{\text {free }}(x) \rightarrow \int d^{4} y S^{\text {free }}(x-y) A(y) S^{\text {free }}(y)$,

and the remaining four propagators in Eq. (11) are replaced with the full quark propagators including the perturbative and nonperturbative parts. Here we use $A_{\mu}(y)=-\frac{1}{2} F_{\mu \nu}(y) y^{\nu}$ where the electromagnetic field strength tensor is written as 
$F_{\mu \nu}(y)=-i\left(\varepsilon_{\mu} q_{\nu}-\varepsilon_{\nu} q_{\mu}\right) e^{i q \cdot y}$. The total perturbative contribution is acquired by performing the replacement mentioned above for the perturbatively interacting quark propagator with the photon and making use of the replacement of the remaining propagators by their free parts.

In the next part, one of the light quark propagators in Eq. (11), defining the photon emission at large distances, is substitute by

$S_{\alpha \beta}^{a b}(x) \rightarrow-\frac{1}{4}\left[\bar{q}^{a}(x) \Gamma_{i} q^{b}(x)\right]\left(\Gamma_{i}\right)_{\alpha \beta}$,

and the rest propagators are substituted with the full quark propagators. Here, $\Gamma_{i}$ represent the full set of Dirac matrices. Once Eq. (16) is plugged into Eq. (11), there appear matrix elements of $\left\langle\gamma(q)\left|\bar{q}(x) \Gamma_{i} q(0)\right| 0\right\rangle$ and $\langle\gamma(q)| \bar{q}(x)$ $\Gamma_{i} G_{\alpha \beta} q(0)|0\rangle$ kinds, representing the nonperturbative contributions. To calculate the nonperturbative contributions, we need these matrix elements which are parameterized in terms of photon wave functions with definite twists. The explicit expressions of the photon DAs are presented in Ref. [43]. The QCD side of the correlation function can be acquired in terms of quark-gluon parameters as well as the DAs of the photon using Eqs. (11)-(16) and after performing the Fourier transformation to remove the calculations to the momentum space. As a result of above procedures the QCD side of the correlation function in terms of the selected structures in momentum space is obtained as

$$
\begin{aligned}
\Pi_{\mu \nu}^{Q C D}(p, q)= & \Pi_{1}^{Q C D} g_{\mu \nu} \not p \phi \phi+\Pi_{2}^{Q C D} g_{\mu \nu} \notin \phi \\
& +\Pi_{3}^{Q C D} q_{\mu} q_{\nu} \notin \phi \\
& +\Pi_{4}^{Q C D}(\varepsilon \cdot p) q_{\mu} q_{\nu} \not p \phi+\cdots,
\end{aligned}
$$

where $\Pi_{i}^{Q C D}$ are functions of the QCD degrees of freedom and photon DAs parameters.

The sum rules are obtained by equating the hadronic and QCD representations of the correlation function. The next step is to perform double Borel transformation $(\mathcal{B})$ over the $p^{2}$ and $(p+q)^{2}$ on the both sides of the sum rules in order to stamp down the contributions of higher states and continuum. To further suppress the contributions of the higher states and continuum we apply the continuum subtraction and use the quark-hadron duality assumption. Hence,

$\mathcal{B} \Pi_{\mu \nu}^{H a d}(p, q)=\mathcal{B} \Pi_{\mu \nu}^{Q C D}(p, q)$,

which leads to

$$
\begin{array}{ll}
\mathcal{B} \Pi_{1}^{H a d}=\mathcal{B} \Pi_{1}^{Q C D}, & \mathcal{B} \Pi_{2}^{\text {Had }}=\mathcal{B} \Pi_{2}^{Q C D}, \\
\mathcal{B} \Pi_{3}^{H a d}=\mathcal{B} \Pi_{3}^{Q C D}, & \mathcal{B} \Pi_{4}^{H a d}=\mathcal{B} \Pi_{4}^{Q C D},
\end{array}
$$

corresponding to the structures $g_{\mu \nu} \not p \notin q, g_{\mu \nu} \notin q, q_{\mu} q_{\nu} \phi q$ and (E.p) $q_{\mu} q_{\nu} \not p q$. By this way we obtain the sum rules for the form factors $F_{1}, F_{2}, F_{3}$ and $F_{4}$, whose explicit expressions are presented in the Appendix.

\section{Numerical analysis}

This section is devoted to the numerical analysis of the electromagnetic multipole moments of the charm-strange $P_{c \bar{s}}$ pentaquarks. We use $m_{u}=m_{d}=0, m_{s}(2 \mathrm{GeV})=$ $0.096_{-0.04}^{+0.08} \mathrm{GeV}, \overline{m_{c}}\left(m_{c}\right)=(1.28 \pm 0.03) \mathrm{GeV}$ (in $\overline{M S}$ scheme) [44], $f_{3 \gamma}(\mu=1 \mathrm{GeV})=-0.0039 \mathrm{GeV}^{2}$ [43], $\langle\bar{u} u\rangle(\mu=1 \mathrm{GeV})=\langle\bar{d} d\rangle(\mu=1 \mathrm{GeV})=(-0.24 \pm$ $0.01)^{3} \mathrm{GeV}^{3}$ [45], $\langle\bar{s} s\rangle(\mu=1 \mathrm{GeV})=0.8\langle\bar{u} u\rangle(\mu=$ $1 \mathrm{GeV}), m_{0}^{2}(\mu=1 \mathrm{GeV})=0.8 \pm 0.1 \mathrm{GeV}^{2},\left\langle g_{s}^{2} G^{2}\right\rangle=$ $0.88 \mathrm{GeV}^{4}$ [12] and $\chi(\mu=1 \mathrm{GeV})=-2.85 \pm 0.5 \mathrm{GeV}^{-2}$ [46]. To obtain a numerical values for the electromagnetic form factors, we need to determine the values of the mass and residue of the $P_{c \bar{s}}$ pentaquarks. The mass and residue of the $P_{c \bar{s}}$ pentaquarks are borrowed from [38]. The parameters entering the photon DAs are presented in Ref. [43].

The estimations for the electromagnetic multipole moments of the charm-strange $P_{c \bar{s}}$ pentaquarks depend on two auxiliary parameters; the continuum threshold $s_{0}$ and Borel mass parameter $M^{2}$. In order to obtain reliable values of the electromagnetic multipole moments from QCD sum rules, we should find the working regions of $s_{0}$ and $M^{2}$ in such a way that the results are insensitive to the variation of these parameters. To obtain a working region for $M^{2}$, we require the pole dominance over the contributions of higher states and continuum. And also the results coming from higher dimensional operators should contribute less than the lower dimensional ones, since operator product expansion (OPE) should be convergent. The above requirements restrict the working region of the Borel parameter to $3 \mathrm{GeV}^{2} \leq M^{2} \leq 5 \mathrm{GeV}^{2}$. The continuum threshold $s_{0}$ is not totally arbitrary and it is relevant to the energy of the first corresponding excited state. In its fixing we again consider the OPE convergence and pole dominance. Our numerical calculations lead to the interval [11-13] $\mathrm{GeV}^{2}$ for this parameter. In Fig. 1, as example, we plot the dependencies of the magnetic dipole moments of the possible pentaquarks on $M^{2}$ at several fixed values of the continuum threshold $s_{0}$. From these graphics we observe that the corresponding magnetic dipole moments seem to be almost independent of $M^{2}$ for different choices of $s_{0}$. However, the dependencies of the obtained results on the continuum threshold are considerable eventhough they are within the limits allowed by the standard prescriptions of the method. We include these variations in the errors of our final results.

Our results for the magnetic dipole and electric quadrupole moments are shown in Table 1. The magnetic octupole moments of the charm-strange $P_{c \bar{s}}$ pentaquarks have also been calculated but they are not presented here because their values are very close to zero. The errors in the results are due to the uncertainties carried by the input parameters and photon DAs as well as those coming from the working win- 

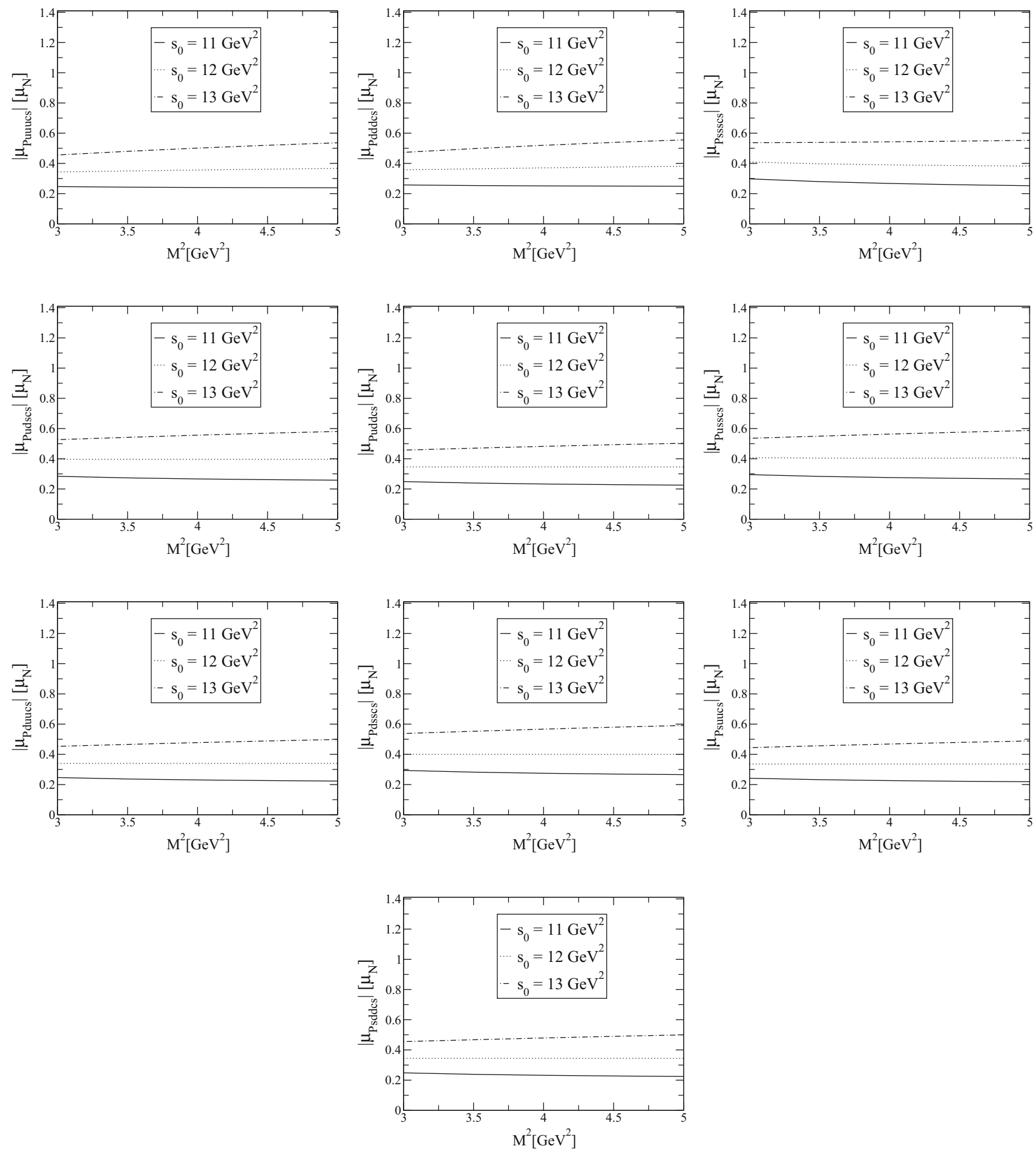

Fig. 1 The magnetic dipole moments for $P_{c \bar{s}}$ pentaquarks versus $M^{2}$ at various fixed values of the $s_{0}$

dows for auxiliary parameters. We should note that the primary source of uncertainties is because of the variations of the results with respect to $s_{0}$. It is worth mentioning that in Table 1 and Fig. 1, the absolute values of the quantities are shown since it is not possible to define the sign of the residue from the mass sum rules. Hence, we cannot predict the signs of the electromagnetic multipole moments. 
Table 1 Numerical values of the magnetic dipole and electric quadrupole moments of $P_{c \bar{s}}$ pentaquarks

\begin{tabular}{|c|c|c|}
\hline State & $\left|\mu_{P_{c s} \mid}\right|\left[\mu_{N}\right]$ & $\left|Q_{P_{c s}}\right|\left[\mathrm{fm}^{2}\right]\left(\times 10^{-3}\right)$ \\
\hline$u d d c \bar{s}$ & $0.36 \pm 0.14$ & $0.21 \pm 0.03$ \\
\hline duис $\bar{s}$ & $0.36 \pm 0.14$ & $0.21 \pm 0.03$ \\
\hline suuc $\bar{s}$ & $0.36 \pm 0.14$ & $0.21 \pm 0.03$ \\
\hline$s d d c \bar{s}$ & $0.36 \pm 0.14$ & $0.21 \pm 0.03$ \\
\hline ииис $\bar{s}$ & $0.39 \pm 0.15$ & $0.22 \pm 0.03$ \\
\hline$d d d c \bar{s}$ & $0.40 \pm 0.15$ & $0.23 \pm 0.04$ \\
\hline $\operatorname{sssc} \bar{s}$ & $0.40 \pm 0.15$ & $0.23 \pm 0.04$ \\
\hline$u d s c \bar{s}$ & $0.42 \pm 0.16$ & $0.23 \pm 0.04$ \\
\hline$u s s c \bar{s}$ & $0.43 \pm 0.16$ & $0.23 \pm 0.04$ \\
\hline$d s s c \bar{s}$ & $0.43 \pm 0.16$ & $0.23 \pm 0.04$ \\
\hline
\end{tabular}

\section{Discussion and concluding remarks}

The electromagnetic multipole moments of the charmstrange $P_{c \bar{s}}$ pentaquarks have been investigated by assuming that these states are represented in diquark-diquarkantiquark picture with quantum numbers $J^{P}=\frac{3}{2}^{-}$. Their magnetic dipole and electric quadrupole moments have been extracted in the framework of light-cone QCD sum rule. The electromagnetic multipole moments of the charm-strange $P_{c \bar{s}}$ pentaquarks are essential dynamical observables, which can contain valuable information of their substructure, charge distribution inside them and their geometric shapes. The numerical values obtained for the magnetic dipole moments are large enough to be measured in future experiments. However we got very small results for the electric quadrupole moments of charm-strange $P_{c \bar{s}}$ pentaquarks indicating a nonspherical charge distribution. As we mentioned above, the values of magnetic octupole moments are obtained to be very close to zero.

Acknowledgements The support of TUBITAK through the Grant no. $115 \mathrm{~F} 183$ is appreciated.

Open Access This article is distributed under the terms of the Creative Commons Attribution 4.0 International License (http://creativecomm ons.org/licenses/by/4.0/), which permits unrestricted use, distribution, and reproduction in any medium, provided you give appropriate credit to the original author(s) and the source, provide a link to the Creative Commons license, and indicate if changes were made.

Funded by SCOAP ${ }^{3}$.

\section{Appendix: QCD sum rules for the electromagnetic form factors $F_{i}$}

The explicit expressions for the electromagnetic form factors $F_{i}$ are given as:

$$
\begin{aligned}
& F_{1}=-\frac{e^{m_{P_{c \bar{s}}}^{2} / M^{2}}}{\lambda_{P_{c \bar{s}}}^{2}}\left\{\frac{e_{S}}{1228800 m_{c}^{2} \pi^{8}}\right. \\
& \times\left[m _ { c } ^ { 5 } \left(-m_{c}^{11} I[-8,5]+5 m_{q_{3}} m_{c}^{10} I[-7,4]\right.\right. \\
& +10 m_{c}^{7}\left(-5 m_{q_{1}} m_{q_{2}} I[-6,4]+I[-6,5]\right) \\
& +50 m_{q_{3}} m_{c}^{6}\left(4 m_{q_{1}} m_{q_{2}} I[-5,3]-I[-5,4]\right) \\
& +10 m_{c}^{5}\left(15 m_{q_{1}} m_{q_{2}} I[-5,4]+2 I[-5,5]\right) \\
& +100 m_{3} m_{c}^{4}\left(6 m_{q_{1}} m_{q_{2}} \times I[-4,3]\right. \\
& +I[-4,4])+15 m_{c}^{3} \\
& \times\left(-10 m_{q_{1}} m_{q_{2}} I[-4,4]+I[-4,5]\right)+75 m_{q_{3}} m_{c}^{2} \\
& \times\left(8 m_{q_{1}} m_{q_{2}} I[-3,3]-I[-3,4]\right) \\
& +m_{c}\left(50 m_{q_{1}} m_{q_{2}} I[-3,4]+4 I[-3,5]\right) \\
& \left.+20 m_{q_{3}}\left(10 m_{q_{1}} m_{q_{2}} I[-2,3]+I[-2,4]\right)\right) \\
& \left.+1600 m_{q_{1}} m_{q_{2}} m_{q_{3}} m_{c} I[0,3]+48 I[0,5]\right] \\
& -\frac{e_{c} m_{c}\left\langle g_{s}^{2} G^{2}\right\rangle}{4718592 \pi^{8}}\left[6 m _ { c } m _ { q _ { 2 3 } } ( m _ { q _ { 1 } } - m _ { q _ { 1 2 } } ) \left(m_{c}^{4} I[-4,2]\right.\right. \\
& \left.-2 m_{c}^{2} I[-3,2]+I[-2,2]\right)+m_{q_{13}}^{2}\left(m_{c}^{7} I[-5,2]\right. \\
& \left.-3 m_{c}^{3} I[-3,2]+2 m_{c} I[-2,2]\right) \\
& +m_{q_{23}}^{2}\left(m_{c}^{7} I[-5,2]-3 m_{c}^{3} I[-3,2]+2 m_{c} I[-2,2]\right) \\
& -2 m_{q_{13}} m_{c}\left(3 ( m _ { q _ { 1 2 } } - m _ { q _ { 2 } } ) \left(m_{c}^{4} I[-4,2]-2 m_{c}^{2} I[-3,2]\right.\right. \\
& +I[-2,2])+m_{q_{23}}\left(m_{c}^{6} I[-5,2]-3 m_{c}^{2} I[-3,2]\right. \\
& +2 I[-2,2]))-2\left(m_{q_{1}}-2 m_{q_{12}}+m_{q_{2}}\right)\left(m_{c}^{6} I[-4,2]\right. \\
& \left.\left.-3 m_{c}^{4} I[-3,2]+3 m_{c}^{2} I[-2,2]-I[-1,2]\right)\right] \\
& +\frac{e_{q_{1}}\left\langle g_{s}^{2} G^{2}\right\rangle}{84934656 m_{c}^{2} \pi^{8}}\left[m _ { c } ^ { 3 } \left(m _ { c } \left(m _ { c } ^ { 2 } \left(31 m_{c}^{6} I[-6,3]\right.\right.\right.\right. \\
& -6 m_{c}^{4}\left(6 m_{q_{23}}^{2}+9 m_{q_{23}} m_{c}+8 m_{q_{3}} m_{c}\right) I[-5,2] \\
& -102 m_{c}^{2} \times I[-4,3]+36\left(3 m_{q_{23}}^{2}+6 m_{q_{23}} m_{c}\right. \\
& \left.\left.+4 m_{q_{3}} m_{c}\right) I[-3,2]-80 I[-3,3]\right) \\
& -24\left(3 m_{q_{23}}^{2}+9 m_{q_{23}} m_{c}+4 m_{q_{3}} m_{c}\right) \\
& \left.\times I[-2,2]-9 I[-2,3])+54 m_{q_{23}} I[-1,2]\right) \\
& -160 I[0,3]]+\frac{e_{q_{2}}\left\langle g_{s}^{2} G^{2}\right\rangle}{84934656 m_{c}^{2} \pi^{8}} \\
& \times\left[m _ { c } ^ { 3 } \left(m _ { c } \left(m _ { c } ^ { 2 } \left(31 m_{c}^{6} I[-6,3]-6 m_{c}^{4}\left(6 m_{q_{13}}^{2}\right.\right.\right.\right.\right. \\
& \left.+9 m_{q_{13}} m_{c}+8 m_{q_{3}} m_{c}\right) I[-5,2]-102 m_{c}^{2} \times I[-4,3] \\
& +36\left(3 m_{q_{13}}^{2}+6 m_{q_{13}} m_{c}+4 m_{q_{3}} m_{c}\right) I[-3,2] \\
& -80 I[-3,3])-24\left(3 m_{q_{13}}^{2}+9 m_{q_{13}} m_{c}+4 m_{q_{3}} m_{c}\right) \\
& \left.\times I[-2,2]-9 I[-2,3])+54 m_{q_{13}} I[-1,2]\right) \\
& -160 I[0,3]]+\frac{e_{q_{3}}\left\langle g_{s}^{2} G^{2}\right\rangle}{5308416 m_{c}^{2} \pi^{8}}\left[m_{c}^{12} I[-6,3]\right. \\
& -6 m_{c}^{8}\left(3\left(m_{q_{12}}^{2}+m_{q_{1}} m_{q_{2}}\right) I[-4,2]+I[-4,3]\right) \\
& +4 m_{c}^{6}\left(9\left(m_{q_{12}}^{2}+m_{q_{1}} m_{q_{2}}\right) \times I[-3,2]-2 I[-3,3]\right) \\
& -3 m_{c}^{4}\left(6\left(m_{q_{12}}^{2}+m_{q_{1}} m_{q_{2}}\right) I[-2,2]+I[-2,3]\right)
\end{aligned}
$$


$-16 I[0,3]]+\frac{e_{q_{12}}\left\langle g_{s}^{2} G^{2}\right\rangle}{42467328 m_{c}^{2} \pi^{8}}$

$\times\left[m_{c}^{3}\left(m_{c}\left(m_{c}^{2}\left(m_{c}^{6} I[-6,3]+3 m_{c}^{4}\left(12 m_{q_{13}} m_{q_{13}}\right.\right.\right.\right.\right.$

$\left.+9 m_{q_{13}} m_{c}+9 m_{q_{23}} m_{c}-16 m_{q_{3}} m_{c}\right)$

$\times I[-5,2]+6 m_{c}^{2} I[-4,3]-36\left(\left(3 m_{q_{23}}-4 m_{q_{3}}\right) m_{c}\right.$

$\left.\left.+3 m_{q_{13}}\left(m_{q_{23}}+m_{c}\right)\right) I[-3,2]+16 I[-3,3]\right)$

$+12\left(6 m_{q_{13}} m_{q_{23}}+9 m_{q_{13}} m_{c}+9 m_{q_{23}} m_{c}-8 m_{q_{3}} m_{c}\right)$

$\left.\times I[-2,2]+9 I[-2,3])-27\left(m_{q_{13}}+m_{q_{23}}\right) I[-1,2]\right)$

$+32 I[0,3]]+\frac{e_{q_{13}} m_{c}\left\langle g_{s}^{2} G^{2}\right\rangle}{4718592 \pi^{8}}$

$\times\left[3\left(m_{q_{13}}-m_{q_{23}}\right) m_{c}^{8} I[-5,2]+2 m_{c}^{5}\left(6 m_{q_{13}} m_{q_{2}}\right.\right.$

$\left.-6 m_{q_{12}} m_{q_{23}}-5 m_{q_{12}} m_{c}+5 m_{q_{2}} m_{c}\right)$

$\times I[-4,2]+12 m_{c}^{3}\left(\left(-2 m_{q_{2}}+m_{q_{23}}\right) m_{c}-m_{q_{13}}\right.$

$\left.\times\left(2 m_{q_{2}}+m_{c}\right)+2 m_{q_{12}}\left(m_{q_{23}}+m_{c}\right)\right) I[-3,2]$

$+6 m_{c}\left(-2 m_{q_{12}} m_{q_{23}}-3 m_{q_{12}} m_{c}+3 m_{q_{2}} m_{c}\right.$

$\left.-2 m_{q_{23}} m_{c}+2 m_{q_{23}}\left(m_{q_{2}}+m_{c}\right)\right) I[-2,2]+\left(4 m_{q_{12}}\right.$

$\left.\left.-3 m_{q_{13}}-4 m_{q_{2}}+3 m_{q_{23}}\right) I[-1,2]\right]+\frac{e_{q_{23}} m_{c}\left\langle g_{s}^{2} G^{2}\right\rangle}{4718592 \pi^{8}}$

$\times\left[3\left(-m_{q_{13}}+m_{q_{23}}\right) m_{c}^{8} I[-5,2]-2 m_{c}^{5}\left(6 m_{q_{12}} m_{q_{13}}\right.\right.$

$\left.-6 m_{q_{1}} m_{q_{23}}-5 m_{q_{1}} m_{c}+5 m_{q_{12}} m_{c}\right)$

$\times I[-4,2]+12 m_{c}^{3}\left(\left(m_{q_{13}}-m_{q_{23}}\right) m_{c}\right.$

$\left.+2 m_{q_{12}}\left(m_{q_{13}}+m_{c}\right)-2 m_{q_{1}}\left(m_{q_{23}}+m_{c}\right)\right) I[-3,2]$

$-6 m_{c}\left(2 m_{q_{12}} m_{q_{13}}-2 m_{q_{1}} m_{q_{23}}-3 m_{q_{1}} m_{c}+3 m_{q_{12}} m_{c}\right.$

$\left.+2 m_{q_{13}} m_{c}-2 m_{q_{23}} m_{c}\right) I[-2,2]-\left(4 m_{q_{1}}\right.$

$\left.\left.-4 m_{q_{12}}-3 m_{q_{13}}+3 m_{q_{23}}\right) I[-1,2]\right]$

$+\frac{e_{s}\left\langle g_{s}^{2} G^{2}\right\rangle}{21233664 \pi^{8}}\left[-4 m_{c}^{8}\left(3\left(m_{q_{13}}-m_{q_{23}}\right)^{2} I[-5,2]\right.\right.$

$\left.+32 I[-5,3]+76 f_{3 \gamma} \pi^{2} I[-5,2] \psi^{a}[u 0]\right)$

$+4 m_{c}^{7}\left(3\left(m_{q_{13}}-m_{q_{23}}\right)^{2} I[-5,2]\right.$

$+3\left(9 m_{q_{1}}-18 m_{q_{12}}+9 m_{q_{2}}-64 m_{q_{3}}\right) I[-4,2]$

$\left.-32 f_{3 \gamma} \pi^{2}\left(m_{q_{13}}+m_{q_{23}}-4 m_{q_{3}}\right) I[-4,1] \psi^{a}\left[u_{0}\right]\right)$

$+8 m_{c}^{6}\left(9\left(8 m_{q_{12}}^{2}+8 m_{q_{1}} m_{q_{2}}+\left(m_{q_{13}}-m_{q_{23}}\right)^{2}\right) I[-4,2]\right.$

$+2 f_{3 \gamma} \pi^{2}\left(3\left(m_{q_{13}}+m_{q_{23}}\right)^{2}\right.$

$\left.\times I[-4,1]-20 I[-4,2]) \psi^{a}\left[u_{0}\right]\right)$

$-3 m_{c}^{5}\left(9\left(3\left(m_{q_{1}}-2 m_{q_{12}}+m_{q_{2}}\right)-16 m_{q_{3}}\right)\right.$

$\times I[-3,2]+4 f_{3 \gamma} \pi^{2}\left(9 m_{q_{1}}+18 m_{q_{12}}-24 m_{q_{13}}\right.$

$\left.\left.+9 m_{q_{2}}-24 m_{q_{23}}+64 m_{q_{3}}\right) I[-3,1] \psi^{a}\left[u_{0}\right]\right)$

$-m_{c}^{4}\left(27\left(32 m_{q_{12}}^{2}+32 m_{q_{1}} m_{q_{2}}+3\left(m_{q_{13}}\right.\right.\right.$

$\left.\left.-m_{q_{23}}\right)^{2}\right) I[-3,2]-128 I[-3,3]$

$+36 f_{3 \gamma} \pi^{2}\left(4\left(8 m_{q_{12}}^{2}+8 m_{q_{1}} m_{q_{2}}\right.\right.$

$\left.\left.-\left(m_{q_{13}}+m_{q_{23}}\right)^{2}\right) I[-3,1]+I[-3,2]\right)$

$\left.\times \psi^{a}\left[u_{0}\right]\right)+3 m_{c}^{3}\left(\left(27\left(m_{q_{1}}-2 m_{q_{12}}+m_{q_{2}}\right)\right.\right.$

$\left.-128 m_{q_{3}}\right) I[-2,2]+24 f_{3 \gamma}\left(3\left(m_{q_{1}}+2 m_{q_{12}}\right.\right.$ $\left.\left.\left.-4 m_{q_{13}}+m_{q_{2}}-4 m_{q_{23}}\right)+16 \pi^{2} m_{q_{3}}\right) I[-2,1] \psi^{a}\left[u_{0}\right]\right)$

$+36 m_{c}^{2}\left(\left(12 m_{q_{12}}^{2}+12 m_{q_{1}} m_{q_{2}}\right.\right.$

$\left.+\left(m_{q_{13}}-m_{q_{23}}\right)^{2}\right) I[-2,2]$

$+2 I l[-2,3]+f_{3 \gamma} \pi^{2}\left(\left(-32 m_{q_{12}}^{2}-32 m_{q_{1}} m_{q_{2}}\right.\right.$

$\left.\left.\left.+3\left(m_{q_{13}}+m_{q_{23}}\right)^{2}\right) I[-2,1]-8 I[-2,2]\right) \psi^{a}\left[u_{0}\right]\right)$

$-192 m_{c}\left(f_{3 \gamma}\left(m_{q_{13}}+m_{q_{23}}\right) I[-1,1] \psi^{a}\left[u_{0}\right]\right)$

$\left.+192\left(f_{3 \gamma}\left(-5\left(m_{q_{13}}+m_{q_{23}}\right)+4 m_{q_{3}}\right) I[0,1] \psi^{a}[u 0]\right)\right]$

$+\frac{e_{s}\left\langle\bar{q}_{1} q_{1}\right\rangle}{18432 m_{c}^{2} \pi^{6}}\left[-6 m_{q_{2}} m_{c}^{5}\left(2 m_{c}^{5} I[-5,3]\right.\right.$

$+6 m_{q_{3}} m_{c}^{4} I[-4,2]+m_{c}^{3}\left(3 m_{0}^{2} I[-4,2]+4 I[-4,3]\right)$

$-6 m_{q_{3}} m_{c}^{2}\left(m_{0}^{2} I[-3,1]+2 I[-3,2]\right)$

$+m_{c}\left(-3 m_{0}^{2} I[-3,2]+2 I[-3,3]\right)$

$\left.+6 m_{q_{3}}\left(I[-2,2]-m_{0}^{2} I[-2,1]\right)\right)$

$-48 m_{q_{2}} I[0,3]+m_{q_{1}}\left(m_{c}^{5}\left(-2 m_{c}^{7} I[-6,3]\right.\right.$

$+3 m_{0}^{2} m_{c}^{5} I[-5,2]+6 m_{q_{3}} m_{c}^{6} I[-5,2]$

$+6 m_{0}^{2} m_{q_{3}} m_{c}^{4} I[-4,1]+6 m_{c}^{3} I[-4,3]$

$-3 m_{0}^{2} m_{c} I[-3,2]-18 m_{q_{3}} m_{c}^{2} I[-3,2]$

$+4 m_{c} I[-3,3]-6 m_{0}^{2} m_{q_{3}} I[-2,1]$

$\left.\left.+12 m_{q_{3}} I[-2,2]\right)+24 m_{0}^{2} m_{q_{3}} m_{c} I[0,1]+8 I[0,3]\right)$

$+4 f_{3 \gamma} \pi^{2}\left(m_{c}^{5}\left(m_{c}\left(m_{q_{1}}\left(3 m_{c}^{4} I[-5,2]\right.\right.\right.\right.$

$+2 m_{c}^{2}\left(-m_{0}^{2}+3 m_{q_{3}} m_{c}\right) I[-4,1]$

$-3 I[-3,2])-6 m_{q_{2}}\left(2 m_{c}^{2} I[-4,2]\right.$

$\left.\left.+m_{0}^{2} I[-3,1]-4 m_{q_{3}} m_{c} I[-3,1]-2 I[-3,2]\right)\right)$

$\left.-6\left(m_{q_{1}}-4 m_{q_{2}}\right) m_{q_{3}} \times I[-2,1]\right)$

$-2 m_{0}^{2}\left(m_{q_{1}}-3 m_{q_{2}}\right) \times m_{q_{3}} m_{c} I[0,0]$

$\left.\left.+2\left(m_{0}^{2}\left(m_{q_{1}}-3 m_{q_{2}}\right)+12 m_{q_{1}} m_{q_{3}} m_{c}\right) I[0,1]\right) \psi^{a}\left[u_{0}\right]\right]$

$-\frac{e_{s} m_{q_{12}}\left\langle\bar{q}_{12} q_{12}\right\rangle}{9216 m_{c}^{2} \pi^{6}}$

$\times\left[2 m_{c}^{12} I[-6,3]-6 m_{q_{3}} m_{c}^{11} I[-5,2]\right.$

$-3 m_{c}^{10}\left(m_{0}^{2} I[-5,2]-4 I[-5,3]\right)-6 m_{q_{3}} m_{c}^{9}\left(m_{0}^{2}\right.$

$\times I[-4,1]-6 I[-4,2])+18 m_{c}^{8}\left(m_{0}^{2} I[-4,2]\right.$

$+I[-4,3])-18 m_{q_{3}} m_{c}^{7}\left(2 m_{0}^{2} I[-3,1]+3 I[-3,2]\right)$

$+m_{c}^{6}\left(-15 m_{0}^{2} I[-3,2]+8 I[-3,3]\right)$

$-6 m_{q_{3}} m_{c}^{5}\left(5 m_{0}^{2} I[-2,1]-4 I[-2,2]\right)$

$-24 m_{0}^{2} m_{q_{3}} m_{c} I[0,1]+40 I[0,3]-4 f_{3 \gamma} \pi^{2}$

$\times\left(3 m_{c}^{10} I[-5,2]+6 m_{q_{3}} m_{c}^{9} I[-4,1]\right.$

$-2 m_{c}^{8}\left(m_{0}^{2} I[-4,1]+6 I[-4,2]\right)$

$+24 m_{q_{3}} m_{c}^{7} \times I[-3,1]+m_{c}^{6}\left(-6 m_{0}^{2} I[-3,1]\right.$

$+9 I[-3,2])+18 m_{q_{3}} m_{c}^{5} I[-2,1]-4 m_{0}^{2} I[0,1]$

$\left.\left.+4 m_{q_{3}} m_{c}\left(m_{0}^{2} I[0,0]+6 I[0,1]\right)\right) \psi^{a}\left[u_{0}\right]\right]$

$+\frac{e_{s}\left\langle\bar{q}_{3} q_{3}\right\rangle}{9216 m_{c}^{2} \pi^{6}}\left[m_{c}^{5}\left(m_{c}^{8} I[-6,3]+3 m_{0}^{2} m_{c}^{6} I[-5,2]\right.\right.$

$-6 m_{c}^{4}\left(3\left(m_{q_{12}}^{2}+m_{q_{1}} m_{q_{2}}\right) I[-4,2]+I[-4,3]\right)$ 


$$
\begin{aligned}
& +m_{c}^{2}\left(18 m_{0}^{2}\left(m_{q_{12}}^{2}+m_{q_{1}} m_{q_{2}}\right) I[-3,1]\right. \\
& \left.-9\left(m_{0}^{2}-4\left(m_{q_{12}}^{2}+m_{q_{1}} m_{q_{2}}\right)\right) I[-3,2]-8 I[-3,3]\right) \\
& +18 m_{0}^{2}\left(m_{q_{12}}^{2}+m_{q_{1}} m_{q_{2}}\right) I[-2,1]+6\left(m_{0}^{2}-3\left(m_{q_{12}}^{2}\right.\right. \\
& \left.\left.\left.+m_{q_{1}} m_{q_{2}}\right)\right) I[-2,2]-3 I[-2,3]\right) \\
& -m_{q_{3}}\left(m _ { c } ^ { 6 } \left(2 m_{c}^{6} I[-6,3]-3 m_{0}^{2} m_{c}^{4} I[-5,2]+6 m_{c}^{2}\right.\right. \\
& \times\left(3\left(m_{q_{12}}^{2}+m_{q_{1}} m_{q_{2}}\right) I[-4,2]-I[-4,3]\right) \\
& +6 m_{0}^{2}\left(m_{q_{12}}^{2}+m_{q_{1}} m_{q_{2}}\right) I[-3,1] \\
& +3\left(m_{0}^{2}-6\left(m_{q_{12}}^{2}+m_{q_{1}} m_{q_{2}}\right)\right) \\
& \times I[-3,2]-4 I[-3,3])+6 m_{0}^{2}\left(m_{q_{12}}^{2}\right. \\
& \left.\left.+m_{q_{1}} m_{q_{2}}\right) I[0,1]-8 I[0,3]\right) \\
& -16 m_{c} I[0,3]+4 f_{3 \gamma} \pi^{2}\left(m _ { c } ^ { 5 } \left(m _ { c } ^ { 2 } \left(2 m_{c}^{4} I[-5,2]\right.\right.\right. \\
& +3 m_{0}^{2} m_{c}^{2} I[-4,1]+12\left(m_{q_{12}}^{2}+m_{q_{1}} m_{q_{2}}\right) \\
& \times I[-3,1]-6 I[-3,2])+m_{q_{3}} m_{c}\left(3 m_{c}^{4} I[-5,2]\right. \\
& -2 m_{0}^{2} m_{c}^{2} I[-4,1]-6\left(m_{q_{12}}^{2}\right. \\
& \left.\left.+m_{q_{1}} m_{q_{2}}\right) I[-3,1]-3 I[-3,2]\right) \\
& -3\left(m_{0}^{2}-4\left(m_{q_{12}}^{2}+m_{q_{1}} m_{q_{2}}\right)\right) \\
& \times I[-2,1]+4 I[-2,2])+3 m_{0}^{2}\left(m_{q_{12}}^{2}\right. \\
& \left.+m_{q_{1}} m_{q_{2}}\right) m_{c} I[0,0]+2\left(-3\left(m_{q_{12}}^{2}+m_{q_{1}} m_{q_{2}}\right)\right. \\
& \left.\left.\left.\left.\times m_{q_{3}}+m_{0}^{2}\left(m_{q_{3}}+6 m_{c}\right)\right) I[0,1]\right) \psi^{a}\left[u_{0}\right]\right]\right\}, \\
& F_{2}=\frac{m_{P_{c \bar{s}}} e^{m_{P_{c \bar{s}}}^{2} / M^{2}}}{\lambda_{P_{c \bar{s}}}^{2}}\left\{\frac{e_{s}\langle\bar{s} s\rangle}{5898240 m_{c}^{2} \pi^{6}}\right. \\
& \times\left[1 6 0 \left(-m_{c}^{5}\left(m_{c}^{7} I[-6,4]-4 m_{q_{3}} m_{c}^{6} I[-5,3]\right.\right.\right. \\
& +3 m_{c}^{3}\left(4\left(m_{q_{12}}^{2}+m_{q_{1}} m_{q_{2}}\right)\right. \\
& \times I[-4,3]-I[-4,4])+12 m_{q_{3}} m_{c}^{2} \\
& \times\left(-3\left(m_{q_{12}}^{2}+m_{q_{1}} m_{q_{2}}\right) I[-3,2]+I[-3,3]\right) \\
& +2 m_{c}\left(6\left(m_{q_{12}}^{2}+m_{q_{1}} m_{q_{2}}\right) I[-3,3]\right. \\
& +I[-3,4])+36 m_{q_{3}}\left(m_{q_{12}}^{2}+m_{q_{1}} m_{q_{2}}\right) I[-2,2] \\
& \left.+8 m_{q_{3}} I[-2,3]\right)-8\left(3 m_{q_{12}}^{2}+3 m_{q_{1}} m_{q_{2}}\right. \\
& \left.\left.+2 m_{q_{3}} m_{c}\right) I[0,3]\right) A\left[u_{0}\right] \\
& +32 \chi\left(m_{c}^{14} I[-7,5]+5 m_{q_{3}} m_{c}^{13} I[-6,4]\right. \\
& +8 m_{c}^{12} I[-6,5]-40 m_{q_{3}} m_{c}^{11} I[-5,4] \\
& +6 m_{c}^{10}\left(5\left(m_{q_{12}}^{2}+m_{q_{1}} m_{q_{2}}\right)\right. \\
& \times I[-5,4]+3 I[-5,5])+30 m_{q_{3}} m_{c}^{9} \\
& \times\left(4\left(m_{q_{12}}^{2}+m_{q_{1}} m_{q_{2}}\right) I[-4,3]+3 I[-4,4]\right) \\
& +4 m_{c}^{8}\left(-15\left(m_{q_{12}}^{2}+m_{q_{1}} m_{q_{2}}\right) I[-4,4]+4 I[-4,5]\right) \\
& +80 m_{q_{3}} m_{c}^{7}\left(3\left(m_{q_{12}}^{2}+m_{q_{1}} m_{q_{2}}\right) I[-3,3]\right. \\
& -I[-3,4])+5 m_{c}^{6}\left(6 \left(m_{q_{12}}^{2}\right.\right. \\
& \left.\left.+m_{q_{1}} m_{q_{2}}\right) I[-3,4]+I[-3,5]\right) \\
& +5 m_{q_{3}} m_{c}^{5}\left(24\left(m_{q_{12}}^{2}+m_{q_{1}} m_{q_{2}}\right) I[-2,3]\right. \\
& +5 I[-2,4])+480\left(m_{q_{12}}^{2}+m_{q_{1}} m_{q_{2}}\right)
\end{aligned}
$$

$\left.\times m_{q_{3}} m_{c} I[0,3]+48 I[0,5]\right) \varphi_{\gamma}\left[u_{0}\right]$

$+5\left(7 m_{c}^{12} I[-6,4]+2\left(9 m_{q_{1}}+18 m_{q_{12}}+6 m_{q_{13}}\right.\right.$

$\left.+9 m_{q_{2}}+6 m_{q_{23}}-32 m_{q_{3}}\right)$

$\times m_{c}^{11} I[-5,3]+6 m_{c}^{10}$

$\times\left(\left(-m_{q_{13}}^{2}+10 m_{q_{13}} m_{q_{2}}+10 m_{q_{1}} m_{q_{23}}-2 m_{q_{13}} m_{q_{23}}\right.\right.$

$\left.-m_{q_{23}}^{2}+10 m_{q_{12}}\left(m_{q_{13}}+m_{q_{23}}\right)\right)$

$\times I[-5,3]+4 I[-5,4])+12 m_{c}^{9}$

$\times\left(3 m_{q_{1}}+6 m_{q_{12}}+8 m_{q_{13}}+3 m_{q_{2}}\right.$

$\left.+8 m_{q_{23}}\right) I[-4,3]-84 m_{c}^{8} I[-4,4]+12 m_{c}^{7}$

$\times\left(9\left(m_{q_{13}}^{2} m_{q_{2}}+2 m_{q_{12}} m_{q_{13}} m_{q_{23}}+m_{q_{1}} m_{q_{23}}^{2}\right) I[-3,2]\right.$

$+\left(3\left(m_{q_{1}}+2 m_{q_{12}}+6 m_{q_{13}}+m_{q_{2}}+6 m_{q_{23}}\right)\right.$

$\left.\left.+16 m_{q_{3}}\right) \times I[-3,3]\right)+2 m_{c}^{6}\left(-9\left(m_{q_{13}}^{2}\right.\right.$

$+6 m_{q_{13}} m_{q_{2}}+6 m_{q_{1}} m_{q_{23}}$

$\left.+2 m_{q_{13}} m_{q_{23}}+m_{q_{23}}^{2}+6 m_{q_{12}}\left(m_{q_{13}}+m_{q_{23}}\right)\right) I[-3,3]$

$+34 I[-3,4])+4 m_{c}^{5}\left(-54\left(m_{q_{13}}^{2} m_{q_{2}}\right.\right.$

$\left.+2 m_{q_{12}} m_{q_{13}} m_{q_{23}}+m_{q_{1}} m_{q_{23}}^{2}\right) I[-2,2]+\left(9 m_{q_{1}}\right.$

$+18 m_{q_{12}}+48 m_{q_{13}}$

$\left.\left.+9 m_{q_{2}}+48 m_{q_{23}}+32 m_{q_{3}}\right) I[-2,3]\right)$

$-3 m_{c}^{4}\left(8\left(m_{q_{13}}\left(2 m_{q_{12}}+m_{q_{13}}+2 m_{q_{2}}\right)\right.\right.$

$\left.+2\left(m_{q_{1}}+m_{q_{12}}+m_{q_{13}}\right) m_{q_{23}}+m_{q_{23}}^{2}\right) I[-2,3]$

$+5 I[-2,4])+6 m_{c}^{3}\left(18\left(m_{q_{13}}^{2} m_{q_{2}}+2 m_{q_{12}} m_{q_{13}} m_{q_{23}}\right.\right.$

$\left.+m_{q_{1}} m_{q_{23}}^{2}\right) I[-1,2]+\left(3 m_{q_{1}}+6 m_{q_{12}}+10 m_{q_{13}}\right.$

$\left.\left.+3 m_{q_{2}}+10 m_{q_{23}}\right) I[-1,3]\right)-48\left(m_{q_{13}}\left(2 m_{q_{12}}\right.\right.$

$\left.+m_{q_{13}}+2 m_{q_{2}}\right)+2\left(m_{q_{1}}+m_{q_{12}}+m_{q_{13}}\right)$

$\left.\times m_{q_{23}}+m_{q_{23}}^{2}\right) \times I[0,3]+16\left(9\left(m_{q_{1}}+2 m_{q_{12}}+4 m_{q_{13}}\right.\right.$

$\left.\left.\left.+m_{q_{2}}+4 m_{q_{23}}\right)+16 m_{q_{3}}\right) m_{c} I[0,3]\right) I_{1}[\tilde{S}]$

$+80\left(m_{c}^{5}\left(-m_{c}^{9} I[-7,4]-4 m_{q_{3}} m_{c}^{8} I[-6,3]\right.\right.$

$+6 m_{c}^{5}\left(4\left(m_{q_{12}}^{2}+m_{q_{1}} m_{q_{2}}\right) I[-5,3]+I[-5,4]\right)$

$+24 m_{q_{3}} m_{c}^{4}\left(3\left(m_{q_{12}}^{2}+m_{q_{1}} m_{q_{2}}\right) I[-4,2]+I[-4,3]\right)$

$+8 m_{c}^{3}\left(6\left(m_{q_{12}}^{2}+m_{q_{1}} m_{q_{2}}\right) I[-4,3]-I[-4,4]\right)$

$+16 m_{q_{3}} m_{c}^{2}\left(-9\left(m_{q_{12}}^{2}+m_{q_{13}}^{2}\right.\right.$

$\left.\left.+m_{q_{23}}^{2}\right)\right) I[-3,2]+2 I[-3,3]+3 m_{c}\left(8\left(m_{q_{12}}^{2}\right.\right.$

$\left.\left.+m_{q_{1}} m_{q_{2}}\right) I[-3,3]+I[-3,4]\right)+12 m_{q_{3}}$

$\left.\times\left(6\left(m_{q_{12}}^{2}+m_{q_{1}} m_{q_{2}}\right) I[-2,2]+I[-2,3]\right)\right)$

$+32\left(3 m_{q_{12}}^{2}+3 m_{q_{1}} m_{q_{2}}\right.$

$\left.\left.\left.\left.+2 m_{q_{3}} m_{c}\right) I[0,3]\right) I_{2}\left[h_{\gamma}\right]\right\}\right\}$,

and

$$
\begin{aligned}
F_{3} & =\frac{4 m_{P_{c \bar{s}}} e^{m_{P_{c \bar{s}}}^{2} / M^{2}}}{\lambda_{P_{c \bar{s}}}^{2}}\left\{\frac { e _ { s } \langle \overline { s } s \rangle } { 7 3 7 2 8 m _ { c } ^ { 2 } \pi ^ { 6 } } \left[4 A\left[u_{0}\right]\right.\right. \\
& \times\left(m _ { c } ^ { 5 } \left(m _ { c } \left(m_{c}^{6} I[-6,3]-3 m_{q_{3}} m_{c}^{5}\right.\right.\right.
\end{aligned}
$$


Table 2 The values of $e_{q_{i}}, e_{q_{i j}}$, $m_{q_{i}}$ and $m_{q_{i j}}$ related to the expressions of the electromagnetic form factors in Eqs. (17)-(19)

\begin{tabular}{lllllllllllll}
\hline$P_{c \bar{s}}$ & $e_{q_{1}}$ & $e_{q_{2}}$ & $e_{q_{3}}$ & $e_{q_{12}}$ & $e_{q_{13}}$ & $e_{q_{23}}$ & $m_{q_{1}}$ & $m_{q_{2}}$ & $m_{q_{3}}$ & $m_{q_{12}}$ & $m_{q_{13}}$ & $m_{q_{23}}$ \\
\hline$u u u c \bar{s}$ & $e_{u}$ & $e_{u}$ & $e_{u}$ & $e_{u}$ & $e_{u}$ & $e_{u}$ & 0 & 0 & 0 & 0 & 0 & 0 \\
$d d d c \bar{s}$ & $e_{d}$ & $e_{d}$ & $e_{d}$ & $e_{d}$ & $e_{d}$ & $e_{d}$ & 0 & 0 & 0 & 0 & 0 & 0 \\
$s s s c \bar{s}$ & $e_{s}$ & $e_{s}$ & $e_{s}$ & $e_{s}$ & $e_{s}$ & $e_{s}$ & $m_{s}$ & $m_{s}$ & $m_{s}$ & $m_{s}$ & $m_{s}$ & $m_{s}$ \\
$u d d c \bar{s}$ & $e_{u}$ & $e_{d}$ & $e_{d}$ & 0 & 0 & $e_{d}$ & 0 & 0 & 0 & 0 & 0 & 0 \\
$u s s c \bar{s}$ & $e_{u}$ & $e_{s}$ & $e_{s}$ & 0 & 0 & $e_{s}$ & 0 & 0 & 0 & 0 & 0 & $m_{s}$ \\
$d u u c \bar{s}$ & $e_{d}$ & $e_{u}$ & $e_{u}$ & 0 & 0 & $e_{u}$ & 0 & 0 & 0 & 0 & 0 & 0 \\
$d s s c \bar{s}$ & $e_{d}$ & $e_{s}$ & $e_{s}$ & 0 & 0 & $e_{s}$ & 0 & 0 & 0 & 0 & 0 & $m_{s}$ \\
$s u u c \bar{s}$ & $e_{s}$ & $e_{u}$ & $e_{u}$ & 0 & 0 & $e_{u}$ & 0 & 0 & 0 & 0 & 0 & 0 \\
$s d d c \bar{s}$ & $e_{s}$ & $e_{d}$ & $e_{d}$ & 0 & 0 & $e_{d}$ & 0 & 0 & 0 & 0 & 0 & 0 \\
$u d s c \bar{s}$ & $e_{u}$ & $e_{d}$ & $e_{s}$ & 0 & 0 & 0 & 0 & 0 & 0 & 0 & 0 & 0 \\
\hline
\end{tabular}

propagators are replaced by their free parts. The values of $e_{q_{i}}, e_{q_{i j}}, m_{q_{i}}$ and $m_{q_{i j}}$ corresponding to different states are given in Table 2.

The functions $I[n, m], I_{1}[\mathcal{A}]$ and $I_{2}[\mathcal{A}]$ are defined as:

$$
\begin{aligned}
I[n, m] & =\int_{m_{c}^{2}}^{s_{0}} d s \int_{m_{c}^{2}}^{s} d l e^{-s / M^{2}} \frac{(s-l)^{m}}{l^{n}} \\
I_{1}[\mathcal{A}] & =\int D_{\alpha_{i}} \int_{0}^{1} d v \mathcal{A}\left(\alpha_{\bar{q}}, \alpha_{q}, \alpha_{g}\right) \delta\left(\alpha_{\bar{q}}+v \alpha_{g}-u_{0}\right), \\
I_{2}[\mathcal{A}] & =\int_{0}^{1} d u A(u) .
\end{aligned}
$$

\section{References}

1. R.L. Jaffe, Multi-quark hadrons. 2. Methods. Phys. Rev. D 15, 281 (1977). https://doi.org/10.1103/PhysRevD.15.281

2. S.K. Choi, Observation of a narrow charmonium-like state in exclusive $B^{ \pm} \rightarrow K^{ \pm} \pi^{+} \pi^{-} J / \psi$ decays. Phys. Rev. Lett. 91, 262001 (2003). https://doi.org/10.1103/PhysRevLett.91.262001. arXiv:hep-ex/0309032

3. R. Aaij, Observation of $J / \psi p$ resonances consistent with pentaquark states in $\Lambda_{b}^{0} \rightarrow J / \psi K^{-} p$ decays. Phys. Rev. Lett. 115, 072001 (2015). https://doi.org/10.1103/PhysRevLett.115.072001. arXiv: 1507.03414

4. R. Faccini, A. Pilloni, A.D. Polosa, Exotic heavy quarkonium spectroscopy: a mini-review. Mod. Phys. Lett. A 27, 1230025 (2012). https://doi.org/10.1142/S021773231230025X. arXiv:1209.0107

5. A. Esposito, A.L. Guerrieri, F. Piccinini, A. Pilloni, A.D. Polosa, Four-quark hadrons: an updated review. Int. J. Mod. Phys. A 30, 1530002 (2015). https://doi.org/10.1142/S0217751X15300021. arXiv: 1411.5997

6. H.-X. Chen, W. Chen, X. Liu, S.-L. Zhu, The hidden-charm pentaquark and tetraquark states. Phys. Rep. 639, 1-121 (2016). https:// doi.org/10.1016/j.physrep.2016.05.004. arXiv:1601.02092

7. A. Ali, J.S. Lange, S. Stone, Exotics: heavy pentaquarks and tetraquarks. Prog. Part. Nucl. Phys. 97, 123-198 (2017). https:// doi.org/10.1016/j.ppnp.2017.08.003. arXiv:1706.00610

8. A. Esposito, A. Pilloni, A.D. Polosa, Multiquark resonances. Phys. Rep. 668,1-97 (2016). https://doi.org/10.1016/j.physrep.2016.11. 002. arXiv: 1611.07920 
9. S.L. Olsen, T. Skwarnicki, D. Zieminska, Non-standard heavy mesons and baryons, an experimental review. Rev. Mod. Phys. 90, 15003 (2018). https://doi.org/10.1103/RevModPhys.90.015003. arXiv: 1708.04012

10. R.F. Lebed, R.E. Mitchell, E.S. Swanson, Heavy-quark QCD exotica. Prog. Part. Nucl. Phys. 93, 143-194 (2017). https://doi.org/10. 1016/j.ppnp.2016.11.003. arXiv:1610.04528

11. F.-K. Guo, C. Hanhart, U.-G. Meißner, Q. Wang, Q. Zhao, B.-S. Zou, Hadronic molecules. Rev. Mod. Phys. 90(1), 015004 (2018). https://doi.org/10.1103/RevModPhys.90.015004. arXiv: 1705.00141

12. M. Nielsen, F.S. Navarra, S.H. Lee, New charmonium states in QCD sum rules: a concise review. Phys. Rep. 497, 41-83 (2010). https://doi.org/10.1016/j.physrep.2010.07.005. arXiv:0911.1958

13. E.S. Swanson, The new heavy mesons: a status report. Phys. Rep. 429, 243-305 (2006). https://doi.org/10.1016/j.physrep.2006.04. 003. arXiv:hep-ph/0601110

14. M.B. Voloshin, Charmonium. Prog. Part. Nucl. Phys. 61, 455-511 (2008). https://doi.org/10.1016/j.ppnp.2008.02.001. arXiv:0711.4556

15. E. Klempt, A. Zaitsev, Glueballs, hybrids, multiquarks. Experimental facts versus QCD inspired concepts. Phys. Rep. 454, 1-202 (2007). https://doi.org/10.1016/j.physrep.2007.07. 006. arXiv:0708.4016

16. S. Godfrey, S.L. Olsen, The exotic XYZ charmonium-like mesons. Ann. Rev. Nucl. Part. Sci. 58, 51-73 (2008). https://doi.org/10. 1146/annurev.nucl.58.110707.171145. arXiv:0801.3867

17. K. Azizi, Y. Sarac, H. Sundu, Possible molecular pentaquark states with different spin and quark configurations. arXiv:1805.06734

18. K. Azizi, Y. Sarac, H. Sundu, Strong decay of $P_{c}(4380)$ pentaquark in a molecular picture. Phys. Lett. B 782, 694-701 (2018). https:// doi.org/10.1016/j.physletb.2018.06.022. arXiv:1802.01384

19. K. Azizi, Y. Sarac, H. Sundu, Hidden bottom pentaquark states with spin 3/2 and 5/2. Phys. Rev. D 96(9), 094030 (2017). https:// doi.org/10.1103/PhysRevD.96.094030. arXiv:1707.01248

20. K. Azizi, Y. Sarac, H. Sundu, Analysis of $P_{c}^{+}(4380)$ and $P_{c}^{+}(4450)$ as pentaquark states in the molecular picture with QCD sum rules. Phys. Rev. D 95(9), 094016 (2017). https://doi.org/10.1103/ PhysRevD.95.094016. arXiv:1612.07479

21. H.-C. Kim, M. Praszalowicz, Magnetic moments of exotic pentaquarks in the chiral quark soliton model. Phys. Lett. B 585, 99-105 (2004). https://doi.org/10.1016/j.physletb.2004.01. 067. arXiv:hep-ph/0308242

22. U. Ozdem, K. Azizi, Magnetic dipole moment of $Z_{b}(10610)$ in light-cone QCD. Phys. Rev. D 97(1), 014010 (2018). https://doi. org/10.1103/PhysRevD.97.014010. arXiv:1709.09714

23. Z.-G. Wang, The magnetic moment of the $Z_{c}(3900)$ as an axialvector tetraquark state with QCD sum rules. Eur. Phys. J. C 78(4), 297 (2018). https://doi.org/10.1140/epjc/ s10052-018-5794-0. arXiv:1712.05664

24. K. Azizi, U. Özdem, The electromagnetic multipole moments of the charged open-flavor $Z_{\bar{c} q}$ states. J. Phys. G 45(5), 055003 (2018). https://doi.org/10.1088/1361-6471/aab56b. arXiv:1802.07711

25. A.K. Agamaliev, T.M. Aliev, M. Savc, Magnetic moment of $X_{Q}$ state with $J^{P C}=1^{+ \pm}$in light cone QCD sum rules. Phys. Rev. D 95(3), 036015 (2017). https://doi.org/10.1103/PhysRevD. 95.036015. arXiv: 1610.03980

26. P.-Z. Huang, W.-Z. Deng, X.-L. Chen, S.-L. Zhu, Magnetic moment of the Theta+ pentaquark state. Phys. Rev. D 69, 074004 (2004). https://doi.org/10.1103/PhysRevD.69.074004. arXiv:hep-ph/0311108

27. Y.R. Liu, P.Z. Huang, W.Z. Deng, X.L. Chen, S.-L. Zhu, Pentaquark magnetic moments in different models. Phys. Rev. C 69, 035205 (2004). https://doi.org/10.1103/PhysRevC.69.035205. arXiv:hep-ph/0312074
28. Z.-G. Wang, W.-M. Yang, S.-L. Wan, Magnetic moment of the pentaquark Theta+(1540) with QCD sum rules. J. Phys. G 31, 703-710 (2005). https://doi.org/10.1088/0954-3899/31/7/015. arXiv:hep-ph/0503073

29. U. Özdem, K. Azizi, Electromagnetic multipole moments of the $P_{c}^{+}(4380)$ pentaquark in light-cone QCD. Eur. Phys. J. C 78(5), 379 (2018). https://doi.org/10.1140/epjc/s10052-018-5873-2. arXiv: 1803.06831

30. Z.-G. Wang, S.-L. Wan, W.-M. Yang, Magnetic moment of the pentaquark Theta+(1540) as diquark-diquark-antiquark state with QCD sum rules. Eur. Phys. J. C 45, 201-209 (2006). https://doi. org/10.1140/epjc/s2005-02400-1. arXiv:hep-ph/0503007

31. G.-J. Wang, R. Chen, L. Ma, X. Liu, S.-L. Zhu, Magnetic moments of the hidden-charm pentaquark states. Phys. Rev. D 94(9), 094018 (2016). https://doi.org/10.1103/PhysRevD.94. 094018. arXiv:1605.01337

32. R. Bijker, M.M. Giannini, E. Santopinto, Magnetic moments of antidecuplet pentaquarks. Phys. Lett. B 595, 260 268 (2004). https://doi.org/10.1016/j.physletb.2004.05.062. arXiv:hep-ph/0403029

33. W.W. Li, Y.R. Liu, P.Z. Huang, W.Z. Deng, X.L. Chen, S.-L. Zhu, Magnetic moments of $\mathrm{J} * * \mathrm{P}=3 / 2+$ pentaquarks. HEPNP 28, 918 (2004). arXiv:hep-ph/0312362

34. U. Ozdem, K. Azizi, Magnetic and quadrupole moments of the $Z_{c}$ (3900). Phys. Rev. D 96(7), 074030 (2017). https://doi.org/10. 1103/PhysRevD.96.074030. arXiv:1707.09612

35. V.L. Chernyak, I.R. Zhitnitsky, B meson exclusive decays into baryons. Nucl. Phys. B 345, 137-172 (1990). https://doi.org/10. 1016/0550-3213(90)90612-H

36. V.M. Braun, I.E. Filyanov, QCD sum rules in exclusive kinematics and pion wave function. Z. Phys. C 44, 157 (1989). https://doi.org/ 10.1007/BF01548594 [Yad. Fiz. 50, 818 (1989)]

37. I.I. Balitsky, V.M. Braun, A.V. Kolesnichenko, Radiative decay $\sigma+\rightarrow p \gamma$ in quantum chromodynamics. Nucl. Phys. B 312, 509550 (1989). https://doi.org/10.1016/0550-3213(89)90570-1

38. Z.-G. Wang, J.-X. Zhang, Possible pentaquark candidates: new excited $\Omega_{c}$ states. Eur. Phys. J. C 78(6), 503 (2018). https://doi. org/10.1140/epjc/s10052-018-5989-4. arXiv:1804.06195

39. V. Pascalutsa, M. Vanderhaeghen, S.N. Yang, Electromagnetic excitation of the Delta(1232)-resonance. Phys. Rep. 437, 125-232 (2007). https://doi.org/10.1016/j.physrep.2006.09.006. arXiv:hep-ph/0609004

40. G. Ramalho, M.T. Pena, F. Gross, Electric quadrupole and magnetic octupole moments of the Delta. Phys. Lett. B 678, 355-358 (2009). https://doi.org/10.1016/j.physletb.2009.06.052. arXiv:0902.4212

41. V.M. Belyaev, B.L. Ioffe, Determination of the baryon mass and baryon resonances from the quantum-chromodynamics sum rule. Strange baryons. Sov. Phys. JETP 57, 716-721 (1983) [Zh. Eksp. Teor. Fiz. 84, 1236 (1983)]

42. I.I. Balitsky, V.M. Braun, Evolution equations for QCD string operators. Nucl. Phys. B 311, 541-584 (1989). https://doi.org/10.1016/ 0550-3213(89)90168-5

43. P. Ball, V.M. Braun, N. Kivel, Photon distribution amplitudes in QCD. Nucl. Phys. B 649, 263-296 (2003). https://doi.org/10.1016/ S0550-3213(02)01017-9. arXiv:hep-ph/0207307

44. C. Patrignani et al., Review of particle physics. Chin. Phys. C 40(10), 100001 (2016). https://doi.org/10.1088/1674-1137/40/10/ 100001

45. B.L. Ioffe, QCD at low energies. Prog. Part. Nucl. Phys. 56, 232-277 (2006). https://doi.org/10.1016/j.ppnp.2005.05.001. arXiv:hep-ph/0502148

46. J. Rohrwild, Determination of the magnetic susceptibility of the quark condensate using radiative heavy meson decays. JHEP 09, 073 (2007). https://doi.org/10.1088/1126-6708/2007/09/073. arXiv:0708.1405 